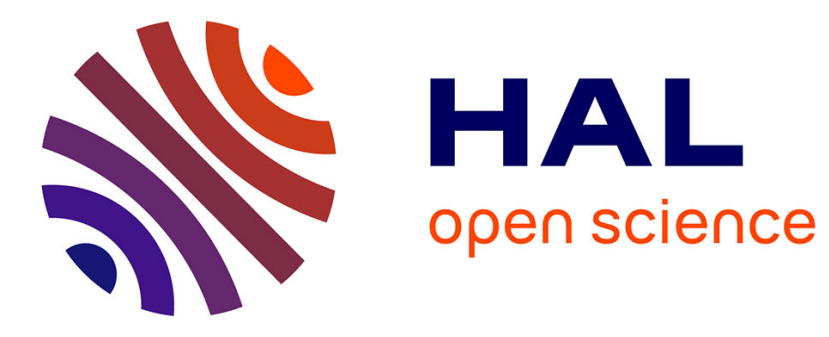

\title{
Some algorithmic results for [2]-sumset covers
}

Laurent Bulteau, Guillaume Fertin, Stéphane Vialette, Roméo Rizzi

\section{To cite this version:}

Laurent Bulteau, Guillaume Fertin, Stéphane Vialette, Roméo Rizzi. Some algorithmic results for [2]-sumset covers. Information Processing Letters, 2015, 115 (1), pp.1-5. hal-01044891

\section{HAL Id: hal-01044891 https://hal.science/hal-01044891}

Submitted on 24 Jul 2014

HAL is a multi-disciplinary open access archive for the deposit and dissemination of scientific research documents, whether they are published or not. The documents may come from teaching and research institutions in France or abroad, or from public or private research centers.
L'archive ouverte pluridisciplinaire HAL, est destinée au dépôt et à la diffusion de documents scientifiques de niveau recherche, publiés ou non, émanant des établissements d'enseignement et de recherche français ou étrangers, des laboratoires publics ou privés. 


\title{
Some algorithmic results for [2]-sumset covers
}

\author{
Laurent Bulteau ${ }^{\mathrm{a}}$, Guillaume Fertin ${ }^{\mathrm{b}}$, Romeo Rizzic ${ }^{\mathrm{c}}$, Stéphane Vialette ${ }^{\mathrm{d}, *}$ \\ ${ }^{a}$ LINA CNRS UMR 6241, Université de Nantes, Nantes, France \\ ${ }^{b}$ LINA CNRS UMR 6241, Université de Nantes, Nantes, France \\ ${ }^{c}$ Dipartimento of Computer Science, Università degli Studi di Verona, Italy \\ ${ }^{d}$ LIGM CNRS UMR 8049, Université Paris-Est Marne-la-Vallée, France
}

\begin{abstract}
Let $X=\left\{x_{i}: 1 \leq i \leq n\right\} \subset \mathbb{N}^{+}$, and $h \in \mathbb{N}^{+}$. The $h$-iterated sumset of $X$, denoted $h X$, is the set $\left\{x_{1}+x_{2}+\ldots+x_{h}: x_{1}, x_{2}, \ldots, x_{h} \in X\right\}$, and the $[h]$ sumset of $X$, denoted $[h] X$, is the set $\bigcup_{i=1}^{h} i X$. A [h]-sumset cover of $S \subset \mathbb{N}^{+}$is a set $X \subset \mathbb{N}^{+}$such that $S \subseteq[h] X$. In this paper, we focus on the case $h=2$, and study the APX-hard problem of computing a minimum cardinality [2]-sumset cover $X$ of $S$ (i.e. computing a minimum cardinality set $X \subset \mathbb{N}^{+}$such that every element of $S$ is either an element of $X$, or the sum of two - non-necessarily distinct - elements of $X$ ). We propose two new algorithmic results. First, we give a fixed-parameter tractable (FPT) algorithm that decides the existence of a [2]-sumset cover of size at most $k$ of a given set $S$. Our algorithm runs in $O\left(2^{(3 \log k-1.4) k}\right.$ poly $\left.(k)\right)$ time, and thus outperforms the $O\left(5^{\frac{k^{2}(k+3)}{2}} k^{2} \log (k)\right)$ time FPT result presented in [Fagnot, Fertin and Vialette, On Finding Small 2-Generating Sets, COCOON 2009, Lecture Notes in Computer Science, Vol. 5609 ]. Second, we show that deciding whether a set $S$ has a smaller [2]-sumset cover than itself is NP-hard.
\end{abstract}

\section{Introduction}

Write $\mathbb{N}^{+}=\{1,2, \ldots\}$ for the set of all natural numbers excluding 0 . Let $X=\left\{x_{i}: 1 \leq i \leq n\right\} \subset \mathbb{N}^{+}$and $h \in \mathbb{N}^{+}$. The $h$-iterated sumset of $X$, denoted $h X$, is the set $\left\{x_{1}+x_{2}+\ldots+x_{h}: x_{1}, x_{2}, \ldots, x_{h} \in X\right\}$, while the $[h]$-sumset of $X$, denoted $[h] X$, is defined as the set $\bigcup_{i=1}^{h} i X$. A $[h]$-sumset cover of $S \subset \mathbb{N}^{+}$ is a set $X \subset \mathbb{N}^{+}$such that $S \subseteq[h] X$. (Notice that we do not require the reverse direction $[h] X \subseteq S$.) The $[h]$-sumset rank of a set $S \subset \mathbb{N}^{+}$is defined to be the smallest cardinality of a $[h]$-sumset cover of $S$. Notice that any set $S \subset \mathbb{N}^{+}$ is a $[h]$-sumset cover of itself for any $h \in \mathbb{N}^{+}$, and hence, in particular, $S$ has

\footnotetext{
* Corresponding author

Email addresses: laurent.bulteau@univ-nantes.fr (Laurent Bulteau), guillaume.fertin@univ-nantes.fr (Guillaume Fertin), romeo.rizzi@univr.it (Romeo Rizzi), vialette@univ-mlv.fr (Stéphane Vialette)
} 
[2]-sumset rank at most $|S|$. A set $S \subset \mathbb{N}^{+}$is said to be [h]-simplifiable if it has a smaller $[h]$-sumset cover than itself.

Example1. Let $S=\{1,2, \ldots, 11\}$. A [2]-sumset cover of $S$ is given by $X=$ $\{1,3,5,6\}$ with [2] $X=\{1,2,3,4,5,6,7,8,9,10,11,12\} \supseteq S$, and hence $S$ is [2]simplifiable since $|X|<|S|$. Furthermore, as there does not exist a smaller [2]-sumset cover of $S$, the [2]-sumset rank of $S$ is $|X|=4$. (Notice that, in this particular example, $X$ is the unique [2]-sumset cover of $S$ of size 4. This is however not a general rule as the set $S=\{1,2, \ldots, 10\}$ has 8 minimum cardinality [2]-sumset covers.)

Example2. Let $S=\{4,7,10\}$. It can be easily checked that $S$ does not have a smaller [2]-sumset cover than itself, and hence that $S$ is not [2]-simplifiable.

In this paper, we focus on the case $h=2$, and thus study the problem of [2]-sumset covering a set $S \subset \mathbb{N}^{+}$with a minimum cardinality set $X \subset \mathbb{N}^{+}$, such that every element of $S$ is either an element of $X$, or the sum of two non-necessarily distinct - elements of $X$. Observe that, in general, a minimum cardinality [2]-sumset cover $X$ does not have to be a subset of $S$ nor intersect it, as shown by the following example: $S=\{4,5,6\}$, whose unique minimum cardinality [2]-sumset cover is $X=\{2,3\}$.

Computing a minimum cardinality [2]-sumset cover of a set of integers is a simple restriction of a natural problem in (algorithmic) number theory [3] which is defined as follows: Given $S \subset \mathbb{N}^{+}$, find a minimum cardinality set $X$ of integers such that every element of $S$ is the sum of a subset of $X$. This problem has been shown to be NP-hard [3], and is related, among other things, to planning radiation therapy (in this setting, elements of $S$ represent radiation dosages required at various points, while an element of $X$ represents a dose delivered simultaneously to multiple points). Other variants, namely the cases in which the elements of $X$ can be negative or fractional, are considered in $[4,11]$.

Computing a minimum cardinality [2]-sumset cover of $\{1,2, \ldots, n\}$ turns out to be a special case of the Postage Stamp problem $[1,17]$. The Postage STAMP problem is defined as follows: for fixed positive integers $h, k \in \mathbb{N}^{+}$, find the largest $n$ for which a $[h]$-sumset cover $X$ of $\{1,2, \ldots, n\}$ of size $k$ exists; this largest $n$ is usually denoted $N(h, k)$. It is easily seen that $N(1, k)=k$ (for $X=\{1,2, \ldots, k\}$ ) and $N(h, 1)=h$ (for $X=\{1\}$ ). Stöhr [13, 14] proved that $N(h, 2)=\left\lfloor\left(h^{2}+6 h+1\right) / 4\right\rfloor$ (Tripathi gives an alternate proof in [17]). Surprisingly enough, no other closed-form expression is known for any other pair $(h, k)$ where one of $h$ and $k$ is fixed [17]. Computing a closed-form expression for $N(2, k)$ (or, going back to our vocabulary, computing a minimum cardinality [2]-sumset cover of $\{1,2, \ldots, n\})$ remains a challenging open problem ; note that an asymptotic bound for $N(2, k)$ is given in [10] (we also refer the reader to Section 4 and Table 1 for a brief discussion on this particular topic).

Computing a minimum cardinality [2]-sumset cover of a set of integers is also related to $\ell$-covering a set of strings $\mathcal{S}$ with a (small) set $\mathcal{C}$ of substrings in $\mathcal{S}$, where $\mathcal{C}$ is said to $\ell$-cover $\mathcal{S}$ if every string in $\mathcal{S}$ can be written as a concatenation 
of at most $\ell$ substrings in $\mathcal{C}$ (see [5] and references therein for a short discussion of applications that arise in the context of computational biology and formal language theory). This string problem is intractable if $\ell=2$ and the set of strings $\mathcal{S}$ is defined over some binary alphabet. In case $\mathcal{S}$ is defined over some unary alphabet with unary notation, the two problems coincide. (Determining the complexity of $\ell$-covering a set of strings $\mathcal{S}$ defined over some unary alphabet with unary notation is, however, open. See Section 4.)

Strongly related to our work are minimum sum covers of finite Abelian groups as investigated in [7, 8] (a subset $X$ of an Abelian group $G$ is a sum cover of $G$ if $\left\{x_{i}+x_{i}: x_{i}, x_{j} \in X\right\}=G$ ). Swanson [15] (see also [9]) gives some constructions and computational results for maximum difference packings of cyclic groups (a subset $X$ of an Abelian group $G$ is a difference packing of $G$ if $\left.|X|(|X|-1)=\left|\left\{g_{i}-g_{j}: g_{i}, g_{j} \in G\right\} \backslash\{0\}\right|\right)$. Haanpää, Huima, and Östergård compute some maximum sum and strict sum packings of cyclic groups are given in [9] (a subset $X$ of an Abelian group $G$ is a sum packing of $G$ if $\left.\left\{x_{i}+x_{i}: x_{i}, x_{j} \in X \wedge x_{i} \neq x_{j}\right\}=G\right)$. Fitch and Jamison [7] give minimum sum and strict sum covers of small cyclic groups, and Wiedemann [18] determines minimum difference covers for cyclic groups of order at most 133 (a subset $X$ of an Abelian group $G$ is a difference cover of $G$ if $\left.\left\{x_{i}-x_{i}: x_{i}, x_{j} \in X\right\}=G\right)$. The best general references are [12] and [16].

Two of the present authors have shown in [6] that computing a minimum cardinality [2]-sumset cover of a set of integers is APX-hard. In the same paper, a representation lemma was given, which implies a fixed-parameter tractable (FPT) algorithm running in $O\left(5^{\frac{k^{2}(k+3)}{2}} k^{2} \log (k)\right)$ time for deciding whether there exists a [2]-sumset cover of cardinality at most $k$ of a set of positive integers $S$. In this paper, we first improve this latter result by providing a new FPT algorithm, whose running time is $O\left(2^{(3 \log k-1.4) k}\right.$ poly $\left.(k)\right)$ (Section 2). We also prove that deciding whether a set $S$ is [2]-simplifiable (i.e., whether $S$ has a smaller [2]-sumset cover than itself), is NP-hard (Section 3).

\section{A faster FPT algorithm for the [2]-sumset cover problem}

In this section, we give an FPT algorithm for deciding whether there exists a [2]-sumset cover of cardinality at most $k$ of a set of positive integers $S$. Its complexity outperforms the one of [6].

We consider graphs that allow for multiple edges ; given a graph $G=(V, E)$, we write $N_{G}(u)$ the (multi-)set of neighbors of vertex $u \in V$. By extension, for any $V^{\prime} \subseteq V, N_{G}\left(V^{\prime}\right)$ denotes the (multi-)set of neighbors of vertices in $V^{\prime}$. Let $S=\left\{s_{i}: 1 \leq i \leq n\right\} \subseteq \mathbb{N}^{+}$, and let $X=\left\{x_{i}: 1 \leq i \leq k\right\}$ be a [2]-sumset cover of $S$. Define an $X$-realization of $S$ to be a bipartite graph $B=(U, V, E)$ equipped with two bijections $\sigma: U \rightarrow S$ and $\chi: V \rightarrow X$ such that $d_{B}(u) \in\{1,2\}$ for all $u \in U$ (where $d_{B}(u)$ stands for the degree of $u$ in $B$ ), and if $d_{B}(u)=1$, say $\{u, v\} \in E$, then $\sigma(u)=\chi(v)$, and if $d_{B}(u)=2$, say $\{u, v\},\left\{u, v^{\prime}\right\} \in E$ (possibly with $v=v^{\prime}$ in case of double edges), then $\sigma(u)=\chi(v)+\chi\left(v^{\prime}\right)$. A $X$-realization $B=(U, V, E)$ of some $S \subset \mathbb{N}^{+}$is said to be minimal if $X$ is a 
minimum cardinality [2]-sumset cover of $S$, i.e., $S$ has [2]-sumset rank $|X|$. The following lemma (see [6], Lemma 10) shows important properties of minimal $X$-realizations in terms of degree and cycle constraints.

Lemma 1 ([6]). Let $B=(U, V, E)$ be a minimal $X$-realization of some $S \subset$ $\mathbb{N}^{+}$. Then, each connected component of $B$ contains a degree-1 vertex $u \in U$, and/or a simple cycle of length $4 \ell+2$ for some $\ell \geq 0$.

To shorten the exposition, we introduce a last definition. A bipartite graph $B=(U, V, E)$ is said to be $k$-reduced if (1) $|U|=|V|=k,(2)$ every vertex $u \in U$ has degree 1 or 2, (3) $B$ contains a perfect matching, and (4) each connected component of $B$ contains a degree-1 vertex $u \in U$, and/or a simple cycle of length $4 \ell+2$ for some $\ell \geq 0$.

Lemma 2. Let $B=(U, V, E)$ be a k-reduced bipartite graph, and $\sigma: U \rightarrow \mathbb{N}^{+}$ be an injective mapping. Then there exists at most one mapping $\chi: V \rightarrow \mathbb{N}^{+}$ such that $\sigma(u)=\sum_{v \in N(u)} \chi(v)$ holds for any $u \in U$, and this mapping can be computed in polynomial-time if it exists.

Proof. We first compute $\chi\left(v^{+}\right)$for exactly one vertex $v^{+} \in V$ in each connected component of $B$. Let $B_{i}=\left(U_{i}, V_{i}, E_{i}\right)$ be any connected component of $B$. If there exists a degree- 1 vertex $u \in U_{i}$, let $v^{+}$be the (only) neighbor of $u$ and set $\chi\left(v^{+}\right)=\sigma(u)$. Otherwise, according to Lemma $1, B_{i}$ contains a simple cycle of length $4 \ell+2$ for some $\ell \geq 0$. Let $\left(u_{1}, v^{+}, u_{2}, v_{2}, \ldots, u_{2 \ell+1}, v_{2 \ell+1}\right)$ be any such cycle, and set $\chi\left(v^{+}\right)=\sum_{i=1}^{2 \ell+1} 1 / 2(-1)^{i-1} \sigma\left(u_{i}\right)$. Clearly, having disposed of this first step, in any connected component $B_{i}=\left(U_{i}, V_{i}, E_{i}\right)$ of $B$ there exists exactly one vertex $v^{+} \in V$ for which $\chi\left(v^{+}\right)$has been set. Next, for any vertex $v \in V$ for which $\chi(v)$ has not been set, consider any path of length $2 p$ leading $v$ to the vertex $v^{+} \in V$ for which $\chi\left(v^{+}\right)$has been set during the first step, say $\left(v, u_{1}, v_{1}, \ldots, v_{p-1}, u_{p}, v^{+}\right)$, and set $\chi(v)=(-1)^{p} \chi\left(v^{+}\right)+\sum_{i=1}^{p}(-1)^{i-1} \sigma\left(u_{i}\right)$. It is now a routine calculation to verify that $\sigma(u)=\sum_{v \in N(u)} \chi(v)$ for any $u \in U$.

Clearly, the above procedure runs in polynomial-time. If $\chi$ has positive, integral values, this is the desired mapping $\chi: V \rightarrow \mathbb{N}^{+}$. Furthermore, by construction, $\chi$ is the only mapping satisfying $\sum_{v \in N(u)} \chi(v)=\sigma(u)$ for any $u \in U$.

Lemma 3. Let $S=\left\{s_{i}: 1 \leq i \leq n\right\} \subset \mathbb{N}^{+}$be a set with [2]-sumset rank $k, X$ be a size-k [2]-sumset cover of $S$, and $B=(U, V, E)$ be a minimal $X$-realization of $S$. Then there exists a subset $U^{\prime} \subseteq U$ of size $k$ such that $B\left[U^{\prime}, V\right]$ is $k$-reduced.

Proof. The proof is constructive: we build a set $U^{\prime}$ as the disjoint union of two sets $U^{0}$ and $U^{1}$ created in the following two steps. In a first step, we start with $U^{0}=\emptyset$, and we consider in turn each connected component $B_{i}=\left(U_{i}, V_{i}, E_{i}\right)$ of $B$. If $B_{i}$ contains a degree- 1 vertex $u \in U_{i}$, add $u$ to $U^{0}$. Otherwise, according to Lemma $1, B_{i}$ contains a simple cycle of size $4 \ell+2$ for some $\ell \geq 0$, say $\left(u_{1}, v_{1}, u_{2}, v_{2}, \ldots, u_{2 \ell+1}, v_{2 \ell+1}\right)$. For every $1 \leq i \leq 2 \ell+1$, add $u_{i}$ to $U^{0}$. Since any vertex $u \in U$ has degree 1 or 2 , at the end of this first step 


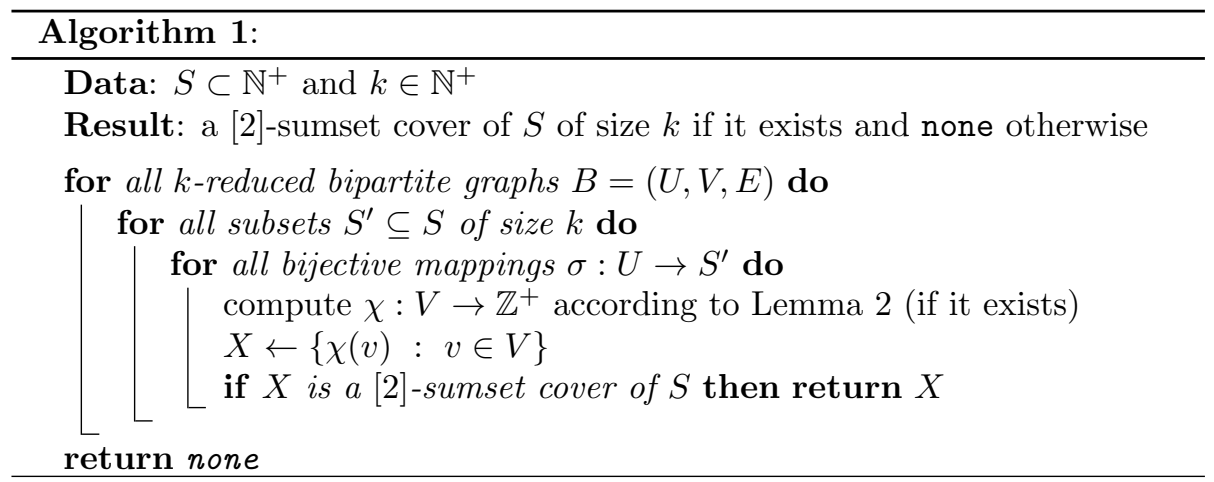

the induced subgraph $B\left[U^{0}, V\right]$ has a perfect matching and $N_{B}\left(U^{0}\right) \cap V_{i} \neq \emptyset$ for each connected component $B_{i}=\left(U_{i}, V_{i}, E_{i}\right)$ of $B$.

For the second step, we again consider in turn each connected component $B_{i}=\left(U_{i}, V_{i}, E_{i}\right)$ of $B$, starting with $U^{1}=\emptyset$. If $V_{i} \subseteq N_{B}\left(U^{0}\right)$ we are done. Otherwise, let $T_{i}$ be any spanning tree of $B_{i}$ rooted at any vertex of $N_{B}\left(U^{0}\right) \cap V_{i}$ (such a vertex does exist since $N_{B}\left(U^{0}\right) \cap V_{i} \neq \emptyset$ ). For any vertex $v \in V_{i} \backslash N_{B}\left(U^{0}\right)$, let $u \in U_{i}$ be its parent in $T_{i}$, and add $u$ to $U^{1}$ ( $u$ exists since $v$ is not the root of $T_{i}$, and $\left.u \notin U^{0}\right)$. Notice that we cannot add twice the same vertex $u$ to $U^{1}$ : this follows from the fact that $T_{i}$ is rooted at some vertex from $V$ and each vertex $u \in U$ has degree at most 2. Then it follows that $U^{0} \cap U^{1}=\emptyset$, and $\left|U^{0} \cup U^{1}\right|=|V|$. It is now a simple matter to check that $B\left[U^{0} \cup U^{1}, V\right]$ is a $k$-reduced graph.

Corollary 4. Let $S \subset \mathbb{N}^{+}$with [2]-sumset rank $k$. Then there exists a subset $S^{\prime} \subseteq S$ of size $k$ of which a $X$-realization is $k$-reduced.

Algorithm 1 is the proposed procedure for finding a minimum [2]-sumset cover of a set $S \subset \mathbb{N}^{+}$with [2]-sumset rank $k$. Correctness of Algorithm 1 follows from Lemma 2 and Corollary 4 . We now turn to evaluating the time complexity of Algorithm 1.

We first focus on the time complexity for enumerating all $k$-reduced graphs. Let $N_{k}$ be the number of $k$-reduced graphs. Recall that every $k$-reduced graph $B=(U, V, E)$ has a perfect matching, and each vertex of $U$ has degree 1 or 2 . It is thus enough to start with a perfect matching of size $k$ and "guess" some degree-2-vertices of $U$. Our approach relies on the following easy lemma.

Lemma 5. Let $B=(U, V, E)$ be a k-reduced graph. Up to a relabeling of $U=$ $\left\{u_{i}: 1 \leq i \leq k\right\}$ and $V=\left\{v_{i}: 1 \leq i \leq k\right\}$, the following properties hold for all $1 \leq i, j \leq n$ : (1) $\left\{u_{i}, v_{i}\right\} \in E$, (2) for any two $u_{i}, u_{j} \in U$, if $d_{B}\left(u_{i}\right)<d_{B}\left(u_{j}\right)$ then $i<j$, and (3) for any $\left\{u_{i}, v_{j}\right\} \in E, j \leq i+1$.

Let $p, 0 \leq p \leq k$, stand for the number of degree- 1 vertices in $U$. According to the above lemma, for each $i, p<i<k$, it is enough to guess the second 
edge of vertex $u_{i}$ among $i+1$ possibilities (another edge connecting $u_{i}$ to $v_{j}$ with $j \leq i+1)$. The number of guesses reduces to $k$ for $i=k$. Then it follows that $N_{k} \leq \sum_{p=0}^{k}\left(\prod_{i=p+1}^{k-1} i+1\right) k=\sum_{p=0}^{k} \frac{k !}{(p+1) !} k \leq k$ !ek since $e=\sum_{p=0}^{\infty} \frac{1}{p !}$. Using the Stirling formula to substitute factorial yields $N_{k}=O\left(k \sqrt{k}\left(\frac{k}{e}\right)^{k}\right)$, and hence $N_{k}=O\left(k^{3 / 2+k} e^{-k}\right)$.

We are now ready to give the total time complexity of Algorithm 1. There are $\left(\begin{array}{l}n \\ k\end{array}\right) k$-subsets $S^{\prime}$ of $S(|S|=n)$, and for each $k$-subset $S^{\prime}$ there are $k$ ! distinct bijective mappings $\sigma: U \rightarrow S^{\prime}$. As for the computation of the mapping $\chi: V \rightarrow$ $X$, this is a poly $(k)$ time procedure (Lemma 2). Besides, checking that solution $X$ is indeed a [2]-sumset cover of $S$ is also a poly $(k)$ time procedure. Combining everything, we obtain $O\left(N_{k}\left(\begin{array}{l}n \\ k\end{array}\right) k ! \operatorname{poly}(k)\right)=O\left(k^{3 / 2+k} e^{-k} \frac{n !}{(n-k) !} \operatorname{poly}(k)\right)=$ $O\left(k^{k} e^{-k} n^{k}\right.$ poly $\left.(k)\right)$. Now remark that, by definition, $k=\Omega(\sqrt{n})$ (since otherwise $X$ could not be a [2]-sumset cover of $S)$, that is $n=O\left(k^{2}\right)$. Hence the overall complexity of Algorithm 1 can be written $O\left(k^{k} e^{-k} k^{2 k}\right.$ poly $\left.(k)\right)$, that is $O\left(2^{(3 \log k-1.4) k} \operatorname{poly}(k)\right)$.

\section{Intractability for [2]-simplification procedures}

Recall that a set $S \subset \mathbb{N}^{+}$is said to be [h]-simplifiable if it has a smaller $[h]$ sumset cover than itself. It is known that computing the smallest cardinality of a [2]-sumset cover of a set $S \subset \mathbb{N}^{+}$(i.e., computing the [2]-sumset rank of $S$ ) is an APX-hard problem [6].

It is a simple matter to see that deciding whether a set $S \subset \mathbb{N}^{+}$is $[h]$ simplifiable is solvable in $O\left((n-1)^{h+1} m^{n-1}\right)$ time, where $n=|S|$ and $m=$ $\max (S)$, where $\max (S)=\max \{s: s \in S\}$. Indeed, this follows from the easy fact that any superset of a $[h]$-sumset cover of $S$ is also an $[h]$-sumset cover of $S$. Therefore, it is enough to check that there exists an $(n-1)$-subset of $[m]$ (there are $\left(\begin{array}{c}m \\ n-1\end{array}\right)=O\left(m^{n-1}\right)$ such subsets) that is a $[h]$-sumset cover of $S$, an easy $O\left((n-1)^{h+1}\right)$ time procedure. This is of course a broad estimation. However, we now prove intractability for any [2]-simplification testing procedure. (In other words, deciding whether the $[\mathbf{2}]$-sumset rank of $S$ is strictly less than $|S|$ is intractable.)

Proposition 6. Deciding whether a set $S \subset \mathbb{N}^{+}$is [2]-simplifiable is NP-hard.

Proof. The reduction is from the Equal Sum Subset of Equal CardiNALITY problem: given a set $T \subset \mathbb{N}^{+}$, are there two disjoint nonempty subsets $A, B \subseteq T$ with $|A|=|B|$ such that $\sum_{a \in A} a=\sum_{b \in B} b$ ? The EQual Sum Subset of Equal Cardinality problem has been shown to be NP-complete in [2].

Let $T=\left\{t_{1}, t_{2}, \ldots t_{n}\right\} \subset \mathbb{N}^{+}$be an arbitrary instance of the EqUAL Sum Subset of Equal Cardinality problem. We take a rather big positive integer $B\left(B=1+\sum_{i=1}^{n} t_{i}\right.$ is actually large enough $)$ and define the set $S=$ $\left\{s_{0}, s_{1}, \ldots, s_{n}\right\} \subset \mathbb{N}^{+}$by $s_{0}=1$ and $s_{i}=2\left(t_{i}+B\right)+1,1 \leq i \leq n$. We claim 
that $S$ is [2]-simplifiable iff the original set $T$ has two disjoint subsets of equal sum and cardinality.

Assume for simplicity that $t_{1}+t_{3}+\ldots+t_{2 k-1}=t_{2}+t_{4}+\ldots+t_{2 k}$ for some $k$. This is clearly equivalent to assuming that $s_{1}+s_{3}+\ldots+s_{2 k-1}=s_{2}+s_{4}+\ldots+s_{2 k}$. Assume further that $t_{2 i-1} \leq t_{2 j-1}$ and $t_{2 i} \geq t_{2 j}$ for all $1 \leq i<j \leq k$. This can be safely assumed through a relabeling of the indices of $t_{1}, t_{3}, \ldots, t_{2 k-1}$ (and of $s_{1}, s_{3}, \ldots, s_{2 k-1}$ ) and a relabeling of the indices of $t_{2}, t_{4}, \ldots, t_{2 k}$ (and of $\left.s_{2}, s_{4}, \ldots, s_{2 k}\right)$. Now, we have that $s_{1}+s_{3}+\ldots+s_{2 q-1} \leq s_{2}+s_{4}+\ldots+s_{2 q}(\dagger)$ for every $1 \leq q \leq k$. Consider now the $2 k$ naturals $d_{0}, d_{1}, d_{2}, \ldots, d_{2 k-1}$ defined by $d_{0}=1$ and $d_{i}=s_{i}-d_{i-1}$ for $1 \leq i \leq 2 k-1$. We claim that each $d_{i}$, $1 \leq i \leq 2 k-1$, is a positive integer. Indeed, for $1 \leq i \leq k-1$, we have $d_{2 i+1}=s_{2 i+1}-d_{2 i}=\left(s_{1}+s_{3}+\ldots+s_{2 i+1}\right)-s_{0}-\left(s_{2}+s_{4}+\ldots+s_{2 i}\right)=$ $2\left(t_{1}+t_{2}+\ldots+t_{2 i+1}\right)+2 B(i+1)+(i+1)-s_{0}-2\left(t_{2}+t_{4}+\ldots+t_{2 i}\right)-2 B i-i=$ $2\left(t_{1}+t_{2}+\ldots+t_{2 i+1}\right)-2\left(t_{2}+t_{4}+\ldots+t_{2 i}\right)+2 B+2>0$ for $B>\sum_{i=1}^{n} t_{i}$, and $d_{2 i}=s_{2 i}-d_{2 i-1}=\left(s_{2}+s_{4}+\ldots+s_{2 i}\right)+s_{0}-\left(s_{1}+s_{3}+\ldots+s_{2 i-1}\right) \geq s_{0}=1$ by $(\dagger)$. Notice that it follows from the above that $d_{2 k-1}=\left(s_{1}+s_{3}+\ldots+\right.$ $\left.s_{2 k-1}\right)-s_{0}-\left(s_{2}+s_{4}+\ldots+s_{2 k-2}\right)$. Combining this with our hypothesis $s_{2 k}=\left(s_{1}+s_{3}+\ldots+s_{2 k-1}\right)-\left(s_{2}+s_{4}+\ldots+s_{k-2}\right)$ yields $s_{2 k}=d_{2 k-1}+s_{0}=$ $d_{2 k-1}+d_{0}$, and hence $s_{i}=d_{i-1}+d_{i \bmod 2 k}$ holds for $1 \leq i \leq 2 k$. Then it follows that $X=\left\{d_{i}: 0 \leq i \leq 2 k-1\right\} \cup\left\{s_{i}: 2 k+1 \leq i \leq n\right\}$ is a [2]-sumset cover of $S$. But $|X|=n-1<n=|S|$, and hence $S$ is 2 -simplifiable.

For the other direction, assume the set $S=\left\{s_{0}, s_{1}, \ldots s_{n}\right\}$ is 2-simplifiable and let $D=\left\{d_{1}, d_{2}, \ldots, d_{k}\right\}, k \leq n$, be a [2]-sumset cover of $S$. Let us represent this situation with a graph $G$ (this graph may have loops). The graph $G$ has $k+1$ vertices labeled $0, d_{1}, \ldots d_{k}$. As for the edges, for every $0 \leq i \leq n$, we have an edge labeled $s_{i}$ between two vertices $d_{p}$ and $d_{q}$ such that $s_{i}=d_{p}+d_{q}$ (if there exists several possibilities for [2]-sumset covering $s_{i}$ with $D$, we choose one arbitrarily). Notice that we have a loop labeled $s_{i}$ if $s_{i}=d_{p}+d_{p}$ for some $d_{p} \in D$, and an edge labeled $s_{i}$ with an endpoint labeled 0 allows us to conveniently represent the case $s_{i}=d_{p}$ as $s_{i}=d_{p}+0, d_{p} \in D$. Since $G$ has $k+1 \leq n+1$ vertices and $n+1$ edges, then $G$ must contain some cycle $C$. Furthermore, the sum of the labels on the edges of $C$ is twice the sum of the labels on the nodes of $C$, and must therefore be an even number. Now, remembering that the $s_{i}$ 's are all odd (by construction from the $t_{i}$ 's) it follows that $C$ is an even cycle, and that the edges of $C$ can be partitioned into two matchings $\mathcal{M}_{1}$ and $\mathcal{M}_{2}$. Notice now that the sum of the labels on the edges of $\mathcal{M}_{1}$ equals the sum of the labels on the edges of $\mathcal{M}_{2}$. From this, and since we chose $B$ big enough, we can exclude the possibility that one edge of $C$ is labeled with $s_{0}=1$. Then it follows that the edges of $\mathcal{M}_{1}$ and the edges of $\mathcal{M}_{2}$ give two disjoint subsets of $T=\left\{t_{1}, t_{2}, \ldots, t_{n}\right\}$ with equal sum and cardinality.

\section{Conclusion and Open Problems}

In this paper, we have studied further the problem of computing a minimum cardinality [2]-sumset cover $X$ of a set $S$ of positive integers. We have provided two new algorithmic results. First, we have proposed an FPT algorithm for 


\begin{tabular}{|c|c|}
\hline $\mathbf{n}$ & {$[\mathbf{2}]$-sumset rank of $\{\mathbf{1}, \mathbf{2}, \ldots\}$} \\
\hline \hline 1,2 & 1 \\
\hline 3,4 & 2 \\
\hline $5,6,7,8$ & 3 \\
\hline $9,10,11,12$ & 4 \\
\hline $13,14,15,16$ & 5 \\
\hline $17,18,19,20$ & 6 \\
\hline $21,22, \ldots, 26$ & 7 \\
\hline $27,28, \ldots, 32$ & 8 \\
\hline $33,34, \ldots, 40$ & 9 \\
\hline $41,42, \ldots, 46$ & 10 \\
\hline $47,48, \ldots, 54$ & 11 \\
\hline $55,56, \ldots, 64$ & 12 \\
\hline $65,66, \ldots, 72$ & 13 \\
\hline
\end{tabular}

Table 1: [2]-sumset rank of $S=\{1,2 \ldots n\}$ for $1 \leq n \leq 72$.

determining whether a set $S$ has a [2]-sumset cover $X$ of cardinality at most $k$ whose complexity outperforms the FPT algorithm from [6]. Second, we have proved that determining whether a set $S$ is [2]-simplifiable is NP-hard.

Though simple to express, the [2]-sumset cover problem is surprisingly hard to tackle. Hence, many open questions remain. We would like to end this paper by mentioning two of them we believe to be the most relevant for algorithmic issues.

First, is the problem of computing the [2]-sumset rank strongly NP-hard ? As mentioned in the introduction, this question is related (and actually was our initial motivation for studying [2]-sumset ranks) to $\ell$-covering a set of strings $\mathcal{S}$ with a (small) set $\mathcal{C}$ of substrings in $\mathcal{S}$, where $\mathcal{C}$ is said to $\ell$-cover $\mathcal{S}$ if every string in $\mathcal{S}$ can be written as a concatenation of at most $\ell$ substrings in $\mathcal{C}$. This problem is intractable if $\ell=2$ and the set of strings $\mathcal{S}$ is defined over some binary alphabet. Proving strongly NP-hardness for computing the [2]-sumset rank of a set of integers would imply intractability if $\ell=2$ and the set of strings $\mathcal{S}$ is defined over some unary alphabet.

Second, focusing on the particular case where $S=\{1,2, \ldots, n\}$, what can be said about minimum [2]-sumset covers of such sets (e.g., in terms of cardinality and structure) ? With practical issues in mind, some experimental results (briefly displayed in Table 1) lead us to formulate the following conjecture:

Conjecture 7. For any positive integer $n \geq 1$, there exists a minimum cardinality [2]-sumset cover $X$ of $\{1,2, \ldots, n\}$ whose maximum element $x_{M}$ satisfies $x_{M} \leq\lceil n / 2\rceil+1$.

By computer simulations, we know this conjecture to be true for any $1 \leq n \leq$ 80. Moreover, for any $n \leq 80$ with $n \notin\{45,46,61,62\}$, there exists a minimum [2]-sumset cover $X$ of $S=\{1,2 \ldots n\}$ whose maximum element $x_{M}$ satisfies 
$x_{M} \leq\lceil n / 2\rceil$. Only in the cases $n \in\{45,46,61,62\}$ do we have $x_{M}>\lceil n / 2\rceil$ for any minimum [2]-sumset cover. In each of these four cases, however, there exists a minimum [2]-sumset cover $X$ of $S$ such that $x_{M}=\lceil n / 2\rceil+1$, hence our above conjecture.

\section{References}

[1] R. Alter and J.A. Barnett. A postage stamp problem. Amer. Math. Montly, 87:206-210, 1980.

[2] M. Cieliebak, S. Eidenbenz, A. Pagourtzis, and K Schlude. On the complexity of variations of equal sum subsets. Nordic J. of Comput., 14(3):151-172, 2008.

[3] M.J. Collins, D. Kempe, J. Saia, and M. Young. Nonnegative integral subset representations of integer sets. IPL, 101(3):129-133, 2007.

[4] M. Develin. Optimal subset representations of integer sets. J. of Number Theo., 89:212-221, 2001.

[5] D.Hermelin, D. Rawitz, R. Rizzi, and S. Vialette. The minimum substring cover problem. Information and Computation, 206(11):1303-1312, 2008.

[6] I. Fagnot, G. Fertin, and S. Vialette. On finding small 2-generating sets. In Proc. 15th Annual International Conference (COCOON), volume 5609 of Lecture Notes in Computer Science, pages 378-387, 2009.

[7] M.A. Fitch and R.E. Jamison. Minimum sum covers of small cyclic groups. Congressus Numerantium, 147:65-81, 2000.

[8] H. Haanpää. Minimum sum and difference covers of abelian groups. J. of Integer Seq., 7(2):Article 04.2.6, 2004.

[9] H. Haanpää, A. Huima, and P.R.J. Östergård. Sets in $\mathbb{Z}_{n}$ with distinct sums of pairs. Discrete App. Math., 138(1-2):99-106, 2004.

[10] L. Moser. On the representation of $1,2, \ldots, n$ by sums. Acta Arith., 6:11$13,1960$.

[11] D. Moulton and D. Petrie. Representing powers of numbers as subset sums of small sets. J. of Number Theo., 89:193-211, 2001.

[12] M.B. Nathanson. Additive Number Theory: the Classical Bases. Number 164 in Graduate Texts in Mathematics. Springer-Verlag, 1996.

[13] A. Stöhr. Gelöste und ungelöste Fragen über Basen der natürlichen Zahlenreihe, i. J. reine Angew. Math., 194:40-65, 1955.

[14] A. Stöhr. Gelöste und ungelöste Fragen über Basen der natürlichen Zahlenreihe, ii. J. reine Angew. Math., 194:111-140, 1955. 
[15] C.N. Swanson. Planar cyclic difference packings. J. of Comb. D esigns, 8:426-434, 2000.

[16] T. Tao and V.H. Vu. Additive Combinatorics, volume 105 of Cambridge studies in advanced mathematics. Cambridge University Press, 2006.

[17] A. Tripathi. A note on the postage stamp problem. Journal of Integer Sequences, 9, 2006. 06.013.

[18] D. Wiedemann. Cyclic difference covers through 133. Congressus Numerantium, 90:181-185, 1992. 